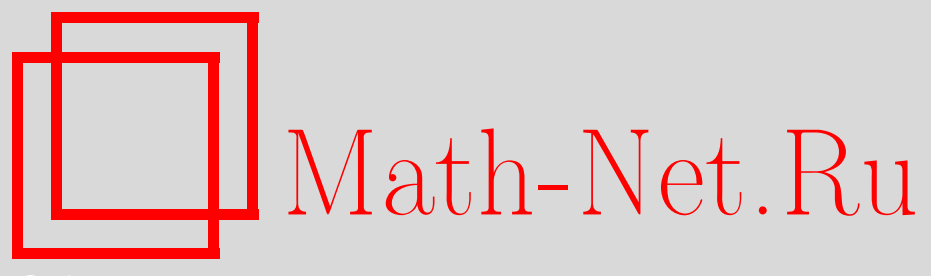

В. В. Напалков, В. Э. Ким, Изоморфизм между пространствами решений дискретного уравнения свертки и уравнения свертки на пространстве целых функций, $M a$ тем. заметки, 2006, том 80, выпуск 5, 733-750

DOI: https://doi.org/10.4213/mzm3082

Использование Общероссийского математического портала Math-Net.Ru подразумевает, что вы прочитали и согласны с пользовательским соглашением http://www . mathnet.ru/rus/agreement

Параметры загрузки:

IP : 54.162 .127 .20

26 апреля 2023 г., 13:26:29

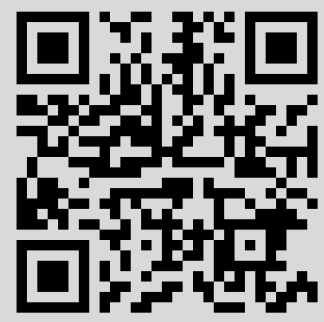




\section{ИЗОМОРФИЗМ МЕЖДУ ПРОСТРАНСТВАМИ РЕШЕНИЙ ДИСКРЕТНОГО УРАВНЕНИЯ СВЕРТКИ И УРАВНЕНИЯ СВЕРТКИ НА ПРОСТРАНСТВЕ ЦЕЛЫХ ФУНКЦИЙ}

\section{В. В. Напалков, В. Э. Ким}

В работе решается задача о восстановлении любого решения однородного уравнения свертки по значениям в целочисленных точках вещественной оси.

Библиография: 18 названий.

Введение. Обозначим через $H(\mathbb{C})$ класс всех целых функций. Для $\rho \in \mathbb{R}, \sigma \in \mathbb{R}$ таких, что $1<\rho<\infty, 0<\sigma<\infty$, рассмотрим в $H(\mathbb{C})$ подклассы: $E[\rho, \sigma]-$ класс целых функций порядка $<\rho$ или порядка $\rho$ и типа $\leqslant \sigma ; E[\rho, \sigma)-$ класс целых функций порядка $<\rho$ или порядка $\rho$ и типа $<\sigma$. Снабдим $E[\rho, \sigma]$ топологией проективного предела банаховых пространств

$$
E_{\rho, \sigma_{j}}=\left\{f \in H(\mathbb{C}):\|f\|_{\rho, \sigma_{j}}=\sup _{z \in \mathbb{C}} \frac{|f(z)|}{\exp \left(\sigma_{j}|z|^{\rho}\right)}<\infty\right\},
$$

где $\left\{\sigma_{j}\right\}_{j \in \mathbb{N}}-$ строго убывающая последовательность чисел из $\mathbb{R}, \lim _{j \rightarrow \infty} \sigma_{j}=\sigma$. Обозначим через $E^{\prime}[\rho, \sigma]$ сильно сопряженное к $E[\rho, \sigma]$ пространство. Пусть $B \in$ $E^{\prime}[\rho, \sigma]$. Функционал $B$ определяет на пространстве $E[\rho, \sigma]$ однородное уравнение свертки

$$
M_{B}[f](z) \equiv\left\langle B_{(\eta)}, f(\eta+z)\right\rangle=0 .
$$

Запись $B_{(\eta)}$ означает, что функционал $B$ действует по переменной $\eta$. Пространство решений уравнения (1) будем обозначать через $W_{B}$.

Уравнения свертки на $E[\rho, \sigma]$ изучались многими математиками (см., например, монографию [1]). В настоящей работе решается задача о восстановлении любого решения уравнения (1) по значениям в целочисленных точках вещественной оси.

Задачи о восстановлении целой функции из какого-либо класса по значениям в целых точках изучались во многих работах. Известно, например, что любую целую функцию $f$ экспоненциального типа $\tau \leqslant \pi$, интегрируемую в квадрате на вещественной оси, можно восстановить по значениям в целых точках с помощью ряда

$$
f(z)=\sum_{n \in \mathbb{Z}} f(n)(-1)^{n} \frac{\sin (\pi z)}{\pi(z-n)}
$$

(C) В.В. НАПАЛков, В.Э. Ким, 2006 
сходящегося равномерно в каждой ограниченной области (см., например, [2; §2.9]).

В общем случае любую функцию из $W_{B}$ восстановить по значениям в целых точках нельзя. В статье найдены достаточные условия, которым должен удовлетворять функционал $B$, для того чтобы эта задача имела решение.

Преобразованием Фурье-Лапласа функционала $B$ называется функция $\widehat{B}(\lambda)=$ $\left\langle B_{(z)}, \exp (i z \lambda)\right\rangle, z, \lambda \in \mathbb{C}$. Положим

$$
\rho^{*}=\frac{\rho}{\rho-1}, \quad \sigma^{*}=\frac{1}{\rho^{*}(\rho \sigma)^{\rho^{*}-1}}, \quad \sigma_{j}^{*}=\frac{1}{\rho^{*}\left(\rho \sigma_{j}\right)^{\rho^{*}-1}}, \quad j \in \mathbb{N} .
$$

Известно (см., например, [3]), что преобразование Фурье-Лапласа устанавливает линейный топологический изоморфизм между пространством $E^{\prime}[\rho, \sigma]$ и пространством $E\left[\rho^{*}, \sigma^{*}\right)$, снабженным топологией индуктивного предела пространств $E_{\rho^{*}, \sigma_{j}^{*}}$. Функция $\widehat{B}(\lambda) \in E\left[\rho^{*}, \sigma^{*}\right)$ называется характеристической функцией уравнения (1).

Обозначим нулевое множество функции $\widehat{B}(\lambda)$ через $\Lambda=\left\{\lambda_{k}\right\}_{k \in \mathbb{N}}$. Будем предполагать, что множество $\Lambda$ удовлетворяет следующим условиям:

(I) все нули $\lambda_{k}, k \in \mathbb{N}$, простые;

(II) $\Lambda \subset\{\lambda \in \mathbb{C}: 0 \leqslant \operatorname{Re} \lambda<2 \pi\}$.

При этом будем предполагать, что $\Lambda$ содержит бесконечно много точек как в верхней, так и в нижней полуплоскости. Разобьем множество $\Lambda$ на две подпоследовательности:

$$
\begin{array}{ll}
\left\{\lambda_{k}^{\prime}\right\}_{k \in \mathbb{N}}=\{\lambda \in \Lambda: \operatorname{Im} \lambda \geqslant 0\}, & \operatorname{Im} \lambda_{k}^{\prime} \nearrow \infty, \\
\left\{\lambda_{k}^{\prime \prime}\right\}_{k \in \mathbb{N}}=\{\lambda \in \Lambda: \operatorname{Im} \lambda<0\}, & \operatorname{Im} \lambda_{k}^{\prime \prime} \searrow-\infty .
\end{array}
$$

Функции $\widehat{B}(\lambda)$ сопоставим целую $2 \pi$-периодическую функцию

$$
\hat{b}(\lambda)=\prod_{\operatorname{Im} \lambda_{k} \geqslant 0}\left(1-\frac{\exp \left(i \lambda_{k}\right)}{\exp (i \lambda)}\right) \prod_{\operatorname{Im} \lambda_{k}<0}\left(1-\frac{\exp (i \lambda)}{\exp \left(i \lambda_{k}\right)}\right) .
$$

Сходимость этих бесконечных произведений и рост функции $\hat{b}(\lambda)$ обсудим позже.

Введем некоторые обозначения. Пусть $V=\left\{v_{k}\right\}_{k \in \mathbb{N}} \subset \mathbb{C},\left|v_{k}\right| \nearrow \infty$. Через $n_{V}(r)$ обозначим число точек из $V$, содержащихся в круге $\{z \in \mathbb{C}:|z| \leqslant r\}$; через $\mu_{V}$ обозначим показатель сходимости [4; с. 19] последовательности $V$; через $N_{V}(r)$ обозначим функцию, определяемую соотношением

$$
N_{V}(r)=\int_{1}^{r} \frac{n_{V}(x)}{x} d x
$$

Сформулируем теперь основной результат статьи.

Теорема 1. Пусть функиионал $B \in E^{\prime}[\rho, \sigma]$ таков, что нулевое множество $\Lambda$ функции $\widehat{B}(\lambda)$ удовлетворяет условиям (I), (II) и условиям

$$
\begin{aligned}
& \lim _{k \rightarrow \infty} \frac{1}{\left|\operatorname{Im} \lambda_{k}^{\prime}\right|^{\rho^{*}}} \ln \left|\prod_{n \neq k}\left(1-\exp \left(i \lambda_{n}^{\prime}-i \lambda_{k}^{\prime}\right)\right)\right|=\tau_{1}<\sigma^{*}, \\
& \lim _{k \rightarrow \infty} \frac{1}{\left|\operatorname{Im} \lambda_{k}^{\prime \prime}\right|^{\rho^{*}}} \ln \left|\prod_{n \neq k}\left(1-\exp \left(i \lambda_{k}^{\prime \prime}-i \lambda_{n}^{\prime \prime}\right)\right)\right|=\tau_{2}<\sigma^{*},
\end{aligned}
$$


где

$$
\begin{aligned}
& \tau_{1}=\varlimsup_{r \rightarrow \infty} \frac{N_{\Omega_{1}}(r)}{(\ln r)^{\rho^{*}}}, \quad \tau_{2}=\varlimsup_{r \rightarrow \infty} \frac{N_{\Omega_{2}}(r)}{(\ln r)^{\rho^{*}}} ; \\
& \Omega_{1}=\left\{\frac{1}{\exp \left(i \lambda_{k}^{\prime}\right)}, k \in \mathbb{N}\right\}, \quad \Omega_{2}=\left\{\exp \left(i \lambda_{k}^{\prime \prime}\right), k \in \mathbb{N}\right\} .
\end{aligned}
$$

Тогда любую функцию $f \in W_{B}$ можно восстановить по значениям на $\mathbb{Z}$ по формуле

$$
f(z)=\sum_{k=1}^{\infty} \frac{\exp \left(i z \lambda_{k}\right)}{\hat{b}_{k}\left(\lambda_{k}\right)} \sum_{n \in \mathbb{Z}} b_{k}(n) f(n),
$$

где $\hat{b}_{k}(\lambda)=\hat{b}(\lambda) /\left(1-\exp \left(i \lambda-i \lambda_{k}\right)\right), b_{k}(n)-$ коэфбициенты ряда Фуръе $\hat{b}_{k}(\lambda)=$ $\sum_{n \in \mathbb{Z}} b_{k}(n) \exp ($ in $\lambda), k \in \mathbb{N}$. Ряд в (5) сходится в топологии пространства $W_{B}$.

Для доказательства теоремы 1 уравнению (1) сопоставим определенное дискретное однородное уравнение свертки. Обозначим через $A$ класс всех комплекснозначных функций, определенных на $\mathbb{Z}$. Введем пространство $A[\rho, \sigma]=\lim _{j} \operatorname{proj} A_{j}$, где

$$
A_{j}=\left\{\psi \in A:\|\psi\|_{A_{j}}=\sup _{n \in \mathbb{Z}} \frac{|\psi(n)|}{\exp \left(\sigma_{j}|n|^{\rho}\right)}<\infty\right\} .
$$

Сопоставим уравнению (1) однородное уравнение свертки на пространстве $A[\rho, \sigma]$ :

$$
\sum_{n \in \mathbb{Z}} b(n) \psi(n+m)=0 \quad \forall m \in \mathbb{Z} .
$$

В этом уравнении неизвестной является функция $\psi \in A[\rho, \sigma]$, а коэффициенты $b(n)$ являются коэффициентами ряда Фурье $\hat{b}(\lambda)=\sum_{n \in \mathbb{Z}} b(n) \exp (i n \lambda)$. Основной метод доказательства теоремы 1 состоит в установлении изоморфизма между пространствами решений уравнений (1) и (6) при определенных условиях на множество $\Lambda$.

1. Уравнение свертки на пространстве $E[\rho, \sigma]$. Очевидно, функции $\exp \left(i z \lambda_{k}\right), k \in \mathbb{N}$, являются решениями уравнения (1). Эти функции называются элементарными решениями уравнения (1). При изучении однородных уравнений свертки возникает следующая задача: можно ли произвольное решение уравнения аппроксимировать элементарными решениями? Эта задача для уравнений свертки на различных пространствах аналитических функций изучалась многими математиками (см., например, [5], [6]). Для однородных уравнений свертки изучается также задача построения в пространстве решений базиса из элементарных решений (см., например, [7]-[9]).

Известно, что любое решение уравнения (1) можно аппроксимировать элементарными решениями (см., например, [10; §7]). А именно, верен следующий результат: $W_{B}=\overline{\operatorname{span}\left\{\exp \left(i z \lambda_{k}\right), k \in \mathbb{N}\right\}}$. Рассмотрим следующий вопрос: можно ли построить в пространстве $W_{B}$ базис из элементарных решений. Известно (см., например, [11]), что существует двойственная связь между задачей построения базиса и задачей интерполяции. Так, из [11] следует, что функции $\exp \left(i z \lambda_{k}\right), k \in \mathbb{N}$, образуют регулярный базис в $\overline{\operatorname{span}\left\{\exp \left(i z \lambda_{k}\right), k \in \mathbb{N}\right\}}$ (а значит, и в пространстве $W_{B}$ ) тогда и только тогда, когда выполняется следующее условие: 
(Q) для любой последовательности $\left\{t_{k}\right\}_{k \in \mathbb{N}} \subset \mathbb{C}$, удовлетворяющей условию

$$
\exists j \in \mathbb{N}: \quad \sup _{k \in \mathbb{N}} \frac{\left|t_{k}\right|}{\left\|\exp \left(i z \lambda_{k}\right)\right\|_{E_{\rho, \sigma_{j}}}}<\infty,
$$

найдется функция $g \in E\left[\rho^{*}, \sigma^{*}\right)$ такая, что $g\left(\lambda_{k}\right)=t_{k} \quad \forall k \in \mathbb{N}$.

Отметим, что

$$
\left\|\exp \left(i z \lambda_{k}\right)\right\|_{E_{\rho, \sigma_{j}}}=\sup _{z \in \mathbb{C}} \frac{\left|\exp \left(i z \lambda_{k}\right)\right|}{\exp \left(\sigma_{j}|z|^{\rho}\right)}=\exp \left(\sigma_{j}^{*}\left|\lambda_{k}\right|^{\rho^{*}}\right) .
$$

Таким образом, условие (7) можно переписать в виде

$$
\exists j \in \mathbb{N}: \quad \sup _{k \in \mathbb{N}} \frac{\left|t_{k}\right|}{\sigma_{j}^{*}\left|\lambda_{k}\right|^{*}}<\infty .
$$

Отметим, что уравнение (1) можно записать в виде дифференциального уравнения бесконечного порядка с постоянными коэффициентами. Рассмотрим ряд Тейлора

$$
\widehat{B}(\lambda)=\sum_{k=0}^{\infty} \frac{c_{k} i^{k}}{k !} \lambda^{k}
$$

Тогда (1) можно переписать в виде

$$
\sum_{k=0}^{\infty} \frac{c_{k}}{k !} f^{(k)}(z)=0
$$

2. Пространство $A[\rho, \sigma]$. Рассмотрим некоторые свойства пространства $A[\rho, \sigma]$. Известно (см., например, [12; гл. 2]), что все вложения $A_{j+1} \subset A_{j}, j \in \mathbb{N}$, являются вполне непрерывными. Это означает, что по терминологии Себаштьяна-иСильвы [13] $A[\rho, \sigma]$ является пространством $\left(M^{*}\right)$. Обозначим через $A^{\prime}[\rho, \sigma]$ сильно сопряженное к $A[\rho, \sigma]$ пространство. Тогда [13; теорема 5$] A^{\prime}[\rho, \sigma]$ является пространством $\left(L N^{*}\right)$. Пространства, сильно сопряженные к $A_{j}, j \in \mathbb{N}$, обозначим через $A_{j}^{\prime}$.

\section{ЛЕмма 1. Имеем}

$$
A_{j}^{\prime}=\left\{\varphi \in A:\|\varphi\|_{A_{j}^{\prime}}=\sum_{n \in \mathbb{Z}}|\varphi(n)| \exp \left(\sigma_{j}|n|^{\rho}\right)<\infty\right\}, \quad j \in \mathbb{N} .
$$

ДокАЗАтЕЛьство. Зафиксируем произвольное $j \in \mathbb{N}$. Определим функции $\Delta_{n} \in A, n \in \mathbb{Z}$, следующим образом: $\Delta_{n}(k)=1$ при $n=k, \Delta_{n}(k)=0$ при $n \neq k$. Очевидно, $\Delta_{n} \in A[\rho, \sigma] \quad \forall n \in \mathbb{Z}$. Пусть $\psi \in A_{j}$. Тогда $\psi(k)=\sum_{n \in \mathbb{Z}} \psi(n) \Delta_{n}(k)$ $\forall k \in \mathbb{Z}$. Функцию $\psi$ можно представить сходящимся в $A_{j}$ рядом $\psi=\sum_{n \in \mathbb{Z}} c_{n} \Delta_{n}$, где $c_{n}=\psi(n), n \in \mathbb{Z}$. Действительно,

$$
\begin{aligned}
\lim _{N \rightarrow \infty}\left\|\psi-\sum_{|n| \leqslant N} c_{n} \Delta_{n}\right\|_{A_{j}} & =\lim _{N \rightarrow \infty} \sup _{k \in \mathbb{Z}} \frac{\left|\psi(k)-\sum_{|n| \leqslant N} \psi(n) \Delta_{n}(k)\right|}{\exp \left(\sigma_{j}|k|^{\rho}\right)} \\
& =\lim _{N \rightarrow \infty} \sup _{|k|>N} \frac{|\psi(k)| \exp \left(\sigma_{j+1}|k|^{\rho}\right)}{\exp \left(\sigma_{j+1}|k|^{\rho}\right) \exp \left(\sigma_{j}|k|^{\rho}\right)} \\
& \leqslant\|\psi\|_{A_{j+1}} \lim _{N \rightarrow \infty} \frac{\exp \left(\sigma_{j+1}|k|^{\rho}\right)}{\exp \left(\sigma_{j}|k|^{\rho}\right)}=0 .
\end{aligned}
$$


Пусть $\varphi \in A_{j}^{\prime}$. Тогда

$$
\langle\varphi, \psi\rangle=\left\langle\varphi, \sum_{n \in \mathbb{Z}} c_{n} \Delta_{n}\right\rangle=\sum_{n \in \mathbb{Z}} c_{n}\left\langle\varphi, \Delta_{n}\right\rangle=\sum_{n \in \mathbb{Z}} \psi(n) \varphi(n),
$$

где $\varphi(n)=\left\langle\varphi, \Delta_{n}\right\rangle$. Таким образом, функционал $\varphi$ можно отождествить с функцией из $A$, т.е. $A_{j}^{\prime} \subset A$. Таким образом, пространство $A_{j}^{\prime}$ имеет вид

$$
A_{j}^{\prime}=\left\{\varphi \in A:\|\varphi\|_{A_{j}^{\prime}}=\sup _{0 \neq \psi \in A_{j}} \frac{|\langle\varphi, \psi\rangle|}{\|\psi\|_{A_{j}}}<\infty\right\} .
$$

Так как

$$
|\langle\varphi, \psi\rangle|=\left|\sum_{n \in \mathbb{Z}} \varphi(n) \psi(n)\right| \leqslant\|\psi\|_{A_{j}} \sum_{n \in \mathbb{Z}}|\varphi(n)| \exp \left(\sigma_{j}|n|^{\rho}\right),
$$

для любой функции $\psi \in A_{j}$, не равной тождественно нулю, получаем

$$
\frac{|\langle\varphi, \psi\rangle|}{\|\psi\|_{A_{j}}} \leqslant \sum_{n \in \mathbb{Z}}|\varphi(n)| \exp \left(\sigma_{j}|n|^{\rho}\right)
$$

Следовательно,

$$
\|\varphi\|_{A_{j}^{\prime}} \leqslant \sum_{n \in \mathbb{Z}}|\varphi(n)| \exp \left(\sigma_{j}|n|^{\rho}\right) .
$$

Определим теперь функцию $\psi_{1}(n) \equiv|\varphi(n)| e^{\sigma_{j}|n|^{\rho}} / \varphi(n)$. Очевидно, $\psi_{1} \in A_{j}$. Тогда

$$
\|\varphi\|_{A_{j}^{\prime}} \geqslant \frac{\left|\left\langle\varphi, \psi_{1}\right\rangle\right|}{\left\|\psi_{1}\right\|_{A_{j}}}=\sum_{n \in \mathbb{Z}}|\varphi(n)| \exp \left(\sigma_{j}|n|^{\rho}\right) .
$$

Сравнивая (9) и (10), заключаем, что $\|\varphi\|_{A_{j}^{\prime}}=\sum_{n \in \mathbb{Z}}|\varphi(n)| \exp \left(\sigma_{j}|n|^{\rho}\right)$.

Из теории счетно-нормированных пространств следует, что $A^{\prime}[\rho, \sigma]=\bigcup_{j \in \mathbb{N}} A_{j}^{\prime}$. Так как функции $\Delta_{n}, n \in \mathbb{Z}$, плотны в $A_{j} \forall j \in \mathbb{N}$, то $A[\rho, \sigma]$ является плотным в каждом из пространств $A_{j}, j \in \mathbb{N}$. Отсюда следует [13], что пространство $A^{\prime}[\rho, \sigma]$ топологически изоморфно индуктивному пределу пространств $A_{j}^{\prime}$.

Лемма 2. Пусть $\lambda \in \mathbb{C}$. Определим функиию $q_{\lambda}(n)=\exp ($ in $\lambda), n \in \mathbb{Z}$. Тогда

$$
\begin{gathered}
\left\|q_{\lambda}\right\|_{A_{j}} \leqslant \exp \left(\sigma_{j}^{*}|\operatorname{Im} \lambda|^{\rho^{*}}\right) \quad \forall \lambda \in \mathbb{C}, \quad \forall j \in \mathbb{N} ; \\
\exp \left(\sigma_{j}^{*}|\operatorname{Im} \lambda|^{\rho^{*}}\right)<\left\|q_{\lambda}\right\|_{A_{j+1}} \quad \forall \lambda \in \mathbb{C}, \quad|\operatorname{Im} \lambda|>r_{0}(j), \quad \forall j \in \mathbb{N} .
\end{gathered}
$$

ДоказАтельство. Зафиксируем произвольные $j \in \mathbb{N}, \lambda \in \mathbb{C}$. Докажем вначале неравенство (11). Имеем

$$
\left\|q_{\lambda}\right\|_{A_{j}}=\sup _{n \in \mathbb{Z}} \frac{|\exp (i n \lambda)|}{\exp \left(\sigma_{j}|n|^{\rho}\right)}=\sup _{n \in \mathbb{Z}} \frac{\exp ((-n) \cdot \operatorname{Im} \lambda)}{\exp \left(\sigma_{j}|n|^{\rho}\right)}=\sup _{\substack{|n|, n \in \mathbb{Z} \\ n \cdot \operatorname{Im} \lambda<0}} \frac{\exp (|n||\operatorname{Im} \lambda|)}{\exp \left(\sigma_{j}|n|^{\rho}\right)} .
$$

Определим для аргумента $r \in \mathbb{R}, r \geqslant 0$, функцию $b_{j, \lambda}(r)=\exp \left(r|\operatorname{Im} \lambda|-\sigma_{j} r^{\rho}\right)$. Нетрудно вычислить, что $\sup _{r \in \mathbb{R}, r \geqslant 0} b_{j, \lambda}(r)=\exp \left(\sigma_{j}^{*}|\operatorname{Im} \lambda|^{\rho^{*}}\right)$ достигается в точке $r_{j, \lambda}=\left(|\operatorname{Im} \lambda| / \rho \sigma_{j}\right)^{\rho^{*}-1}$. Обозначим через $n_{j, \lambda}$ точку из $\mathbb{Z}$, удовлетворяющую условиям: 
1) $b_{j, \lambda}\left(\left|n_{j, \lambda}\right|\right)=\sup _{r \in \mathbb{Z}, r \geqslant 0} b_{j, \lambda}(r)$;

2) $n_{j, \lambda} \cdot \operatorname{Im} \lambda<0$ (если этим условиям удовлетворяют сразу две точки (слева и справа от $\left.r_{j, \lambda}\right)$, то можно выбрать любую из них).

Тогда $\left\|q_{\lambda}\right\|_{A_{j}}=b_{j, \lambda}\left(\left|n_{j, \lambda}\right|\right) \leqslant b_{j, \lambda}\left(r_{j, \lambda}\right)=\exp \left(\sigma_{j}^{*}|\operatorname{Im} \lambda|^{\rho^{*}}\right)$, т.е. выполняется (11).

Докажем теперь (12). Очевидно, для точки $n_{j, \lambda}$ возможны два варианта:

а) $r_{j, \lambda}-1<\left|n_{j, \lambda}\right| \leqslant r_{j, \lambda}$

б) $r_{j, \lambda} \leqslant\left|n_{j, \lambda}\right|<r_{j, \lambda}+1$.

Допустим, выполняется вариант а). Тогда

$$
\left\|q_{\lambda}\right\|_{A_{j+1}}=\|\exp (\operatorname{in} \lambda)\|_{A_{j+1}}=b_{j+1, \lambda}\left(\left|n_{j+1, \lambda}\right|\right)>b_{j+1, \lambda}\left(r_{j+1, \lambda}-1\right) .
$$

Нетрудно вычислить, что $b_{j+1, \lambda}\left(r_{j+1, \lambda}-1\right)=\exp \left(\alpha_{\lambda} \sigma_{j+1}^{*}|\operatorname{Im} \lambda|^{\rho^{*}}\right)$, где

$$
\alpha_{\lambda}=\rho^{*}-\rho^{*}\left(\frac{\rho \sigma_{j+1}}{|\operatorname{Im} \lambda|}\right)^{\rho^{*}-1}-\left(\rho^{*}-1\right)\left(1-\left(\frac{\rho \sigma_{j+1}}{|\operatorname{Im} \lambda|}\right)^{\rho^{*}-1}\right)^{\rho} .
$$

Заметим, что $\alpha_{\lambda} \rightarrow 1$ при $|\operatorname{Im} \lambda| \rightarrow \infty$. Следовательно, найдется число $r_{0}(j)$ такое, что $\alpha_{\lambda} \sigma_{j+1}^{*}>\sigma_{j}^{*}$ при $|\operatorname{Im} \lambda|>r_{0}(j)$. Таким образом, $b_{j+1, \lambda}\left(r_{j+1, \lambda}-1\right)>$ $\exp \left(\sigma_{j}^{*}|\operatorname{Im} \lambda|^{\rho^{*}}\right)$ при $|\operatorname{Im} \lambda|>r_{0}(j)$. Следовательно, выполняется (12). Аналогичным способом доказывается неравенство (12) и в случае б).

3. Преобразования Фурье-Лапласа и Меллина. Рассмотрим в $H(\mathbb{C})$ подкласс $P(\mathbb{C})=\{g \in H(\mathbb{C}): g(\lambda)=g(\lambda+2 \pi) \forall \lambda \in \mathbb{C}\}$. Для каждого $j \in \mathbb{N}$ рассмотрим банахово пространство

$$
P_{j}=\left\{g \in P(\mathbb{C}):\|g\|_{P_{j}}=\sup _{\lambda \in \mathbb{C}} \frac{|g(\lambda)|}{\exp \left(\sigma_{j}^{*}|\operatorname{Im} \lambda|^{\rho^{*}}\right)}<\infty\right\} .
$$

Определим пространство $P\left[\rho^{*}, \sigma^{*}\right)=\lim _{j}$ ind $P_{j}$. Введем отображение $L$, которое каждому функционалу $\varphi \in A^{\prime}[\rho, \sigma]$ ставит в соответствие его преобразование ФурьеЛапласа $\widehat{\varphi}(\lambda)=\langle\varphi, \exp ($ in $\lambda)\rangle=\sum_{n \in \mathbb{Z}} \varphi(n) \exp ($ in $\lambda), \lambda \in \mathbb{C}$.

ТЕОРема 2. Отображение L устанавливает линейный топологический изоморбизм между пространствами $A^{\prime}[\rho, \sigma]$ и $P\left[\rho^{*}, \sigma^{*}\right)$.

Доказательство. 1) Пусть $\varphi \in A^{\prime}[\rho, \sigma]$. Тогда найдется $j \in \mathbb{N}$ такое, что $\|\varphi\|_{A_{j}^{\prime}}<\infty$. С учетом неравенства (11) и леммы 1 для любого $\lambda \in \mathbb{C}$ получаем оценку

$$
\sum_{n \in \mathbb{Z}}\left|\varphi(n) e^{i n \lambda}\right| \leqslant \sup _{n \in \mathbb{Z}} \frac{|\exp (i n \lambda)|}{\exp \left(\sigma_{j}|n|^{\rho}\right)} \sum_{n \in \mathbb{Z}}|\varphi(n)| \exp \left(\sigma_{j}|n|^{\rho}\right) \leqslant\|\varphi\|_{A_{j}^{\prime}} \exp \left(\sigma_{j}^{*}|\operatorname{Im} \lambda|^{\rho^{*}}\right) .
$$

Из (13) следует, что ряд $\sum_{n \in \mathbb{Z}} \varphi(n) \exp (i n \lambda)$ сходится абсолютно и равномерно в круге $\{\lambda \in \mathbb{C}:|\lambda| \leqslant R\} \quad \forall R>0$. Следовательно, $\widehat{\varphi} \in H(\mathbb{C})$. Тогда, очевидно, $\widehat{\varphi} \in P(\mathbb{C})$. Кроме того, из (13) следует, что

$$
\|\widehat{\varphi}\|_{P_{j}} \leqslant\|\varphi\|_{A_{j}^{\prime}}<\infty
$$


Из (14) следует, что $\widehat{\varphi} \in P\left[\rho^{*}, \sigma^{*}\right)$ и что отображение $L: A^{\prime}[\rho, \sigma] \rightarrow P\left[\rho^{*}, \sigma^{*}\right)$ является непрерывным.

2) Пусть $g \in P\left[\rho^{*}, \sigma^{*}\right)$. Тогда $\|g\|_{P_{j}}<\infty$ при некотором $j \in \mathbb{N}$. Для $\lambda \in \mathbb{C}$ обозначим $x=\operatorname{Re} \lambda, y=\operatorname{Im} \lambda$. Так как $g \in P(\mathbb{C})$, то (см., например, [14; гл. 7, §5]) функция $g$ единственным образом раскладывается в ряд Фурье:

$$
g(\lambda)=\sum_{n \in \mathbb{Z}} a_{n} \exp (\text { in } \lambda), \quad a_{n}=\frac{1}{2 \pi} \int_{0}^{2 \pi} \frac{g(\lambda)}{\exp (\text { in } \lambda)} d x .
$$

Введем функцию $a \in A$ такую, что $a(n)=a_{n} \forall n \in \mathbb{Z}$. Очевидно, выполняется оценка $|g(\lambda)| \leqslant\|g\|_{P_{j}} \exp \left(\sigma_{j}^{*}|y|^{\rho^{*}}\right) \quad \forall \lambda \in \mathbb{C}$. Таким образом,

$$
|a(n)|=\left|\frac{1}{2 \pi} \int_{0}^{2 \pi} \frac{g(\lambda)}{\exp (i n \lambda)} d x\right| \leqslant\|g\|_{P_{j}} \exp \left(\sigma_{j}^{*}\left(|y|^{\rho^{*}}+n y\right)\right) \quad \forall n \in \mathbb{Z}, \quad \forall y \in \mathbb{R} .
$$

Следовательно,

$$
|a(n)| \leqslant\|g\|_{P_{j}} \inf _{y \in \mathbb{R}} \exp \left(\sigma_{j}^{*}\left(|y|^{\rho^{*}}+n y\right)\right)
$$

Так как

$$
\inf _{y \in \mathbb{R}} \exp \left(\sigma_{j}^{*}\left(|y|^{\rho^{*}}+n y\right)\right)=\exp \left(-\sigma_{j}|n|^{\rho}\right)
$$

то

$$
|a(n)| \exp \left(\sigma_{j+1}|n|^{\rho}\right) \leqslant\|g\|_{P_{j}} \exp \left(\left(\sigma_{j+1}-\sigma_{j}\right)|n|^{\rho}\right) \quad \forall n \in \mathbb{Z} .
$$

Отсюда получаем

$$
\|a\|_{A_{j+1}^{\prime}}=\sum_{n \in \mathbb{Z}}|a(n)| \exp \left(\sigma_{j+1}|n|^{\rho}\right) \leqslant\|g\|_{P_{j}} \sum_{n \in \mathbb{Z}} \exp \left(\left(\sigma_{j+1}-\sigma_{j}\right)|n|^{\rho}\right) .
$$

Обозначим через $C_{j}$ сумму сходящегося ряда $\sum_{n \in \mathbb{Z}} \exp \left(\left(\sigma_{j+1}-\sigma_{j}\right)|n|^{\rho}\right)$. Тогда

$$
\|a\|_{A_{j+1}^{\prime}} \leqslant C_{j}\|g\|_{P_{j}}<\infty
$$

Из (15) следует, что $a \in A^{\prime}[\rho, \sigma]$, причем $\hat{a}(\lambda) \equiv g(\lambda)$. Кроме того, из (15) следует, что отображение $L^{-1}: P\left[\rho^{*}, \sigma^{*}\right) \rightarrow A^{\prime}[\rho, \sigma]$ является непрерывным.

Обозначим через $H_{0}$ множество всех аналитических в $\mathbb{C} \backslash\{0\}$ функций. Определим пространство $H\left[\rho^{*}, \sigma^{*}\right)=\lim _{j}$ ind $H_{j}$, где

$$
H_{j}=\left\{f \in H_{0}:\|f\|_{H_{j}}=\sup _{\xi \in \mathbb{C} \backslash\{0\}} \frac{|f(\xi)|}{\exp \left(\sigma_{j}^{*}|\ln | \xi||^{*}\right)}<\infty\right\} .
$$

Пусть $\varphi \in A^{\prime}[\rho, \sigma]$. Тогда по теореме 2 функция $\widehat{\varphi}(\lambda)=\sum_{n \in \mathbb{Z}} \varphi(n) \exp ($ in $\lambda)$ принадлежит $P_{j} \subset P\left[\rho^{*}, \sigma^{*}\right)$ при некотором $j \in \mathbb{N}$. При замене переменной $\lambda=-i \operatorname{Ln} \xi$, $\xi \in \mathbb{C} \backslash\{0\}$, функция $\widehat{\varphi}(\lambda)$ перейдет в функцию $\widetilde{\varphi}(\xi)=\sum_{n \in \mathbb{Z}} \varphi(n) \xi^{n}=\left\langle\varphi, \xi^{n}\right\rangle$. Очевидно, $\widetilde{\varphi} \in H_{j} \subset H\left[\rho^{*}, \sigma^{*}\right)$, причем $\|\widehat{\varphi}\|_{P_{j}}=\|\widetilde{\varphi}\|_{H_{j}}$. Функция $\widetilde{\varphi}$ называется преобразованием Меллина функционала $\varphi$.

Пусть теперь $f \in H_{j} \subset H\left[\rho^{*}, \sigma^{*}\right)$ при некотором $j \in \mathbb{N}$. Функцию $f$ можно единственным образом разложить в ряд Лорана $f(\xi)=\sum_{n \in \mathbb{Z}} b_{n} \xi^{n}$. При замене переменной $\xi=\exp (i \lambda)$ функция $f(\xi)$ перейдет в функцию $f^{*}(\lambda)=\sum_{n \in \mathbb{Z}} b_{n} \exp (i n \lambda)$. 
Очевидно, $f^{*} \in P\left[\rho^{*}, \sigma^{*}\right)$, причем $\left\|f^{*}\right\|_{P_{j}}=\|f\|_{H_{j}}$. Введем функцию $b \in A$ такую, что $b(n)=b_{n} \forall n \in \mathbb{Z}$. По теореме $2 b \in A^{\prime}[\rho, \sigma], \hat{b}(\lambda) \equiv f^{*}(\lambda)$. Кроме того, очевидно, $f(\xi) \equiv \tilde{b}(\xi)$. Таким образом, мы получили следующее утверждение.

Теорема 3. Отображение $\varphi \rightarrow \widetilde{\varphi}$ устанавливает линейный топологический изомоморфизм между пространствами $A^{\prime}[\rho, \sigma]$ u $H\left[\rho^{*}, \sigma^{*}\right)$.

4. Сходимость бесконечных произведений. Пусть $f \in H(\mathbb{C}), p>1$. Обозначим

$$
\tau_{f}(p):=\varlimsup_{r \rightarrow \infty}\left(\frac{\ln \max _{|z|=r}|f(z)|}{(\ln r)^{p}}\right) .
$$

Если $\tau_{f}(p)<\infty$ и $V=\left\{v_{k}\right\}_{k \in \mathbb{N}}-$ нулевое множество $f$, то [15; теорема 4] выполняется

$$
\tau_{f}(p)=\varlimsup_{r \rightarrow \infty} \frac{N_{V}(r)}{(\ln r)^{p}} .
$$

Лемма 3. Пусть $G(\lambda) \in P[p, q), p>1,0<q<\infty, S=\left\{s_{k}\right\}_{k \in \mathbb{N}},\left|\operatorname{Im} s_{k}\right| \nearrow \infty,-$ нули $G(\lambda)$, содержашиеся в полосе $\{z \in \mathbb{C}: 0 \leqslant \operatorname{Re} z<2 \pi\}$ (кажсый нуль выписъвается столъко раз, какова его кратность). Тогда функция

$$
Q(\lambda)=\prod_{\operatorname{Im} s_{k} \geqslant 0}\left(1-\frac{\exp \left(i s_{k}\right)}{\exp (i \lambda)}\right) \prod_{\operatorname{Im} s_{k}<0}\left(1-\frac{\exp (i \lambda)}{\exp \left(i s_{k}\right)}\right)
$$

принадлежит пространству $P[p, \delta)$, где $\delta=e(\sqrt{2})^{2 p-1} q \pi$.

ДокАзАтЕЛЬство. Нулевым множеством $G(\lambda)$ является множество $S_{G}=$

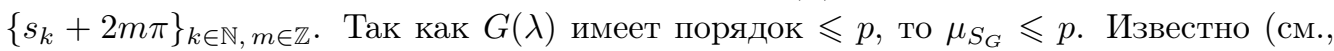
например, [12; гл. 1]), что $\mu_{S_{G}}=\mu_{S}+1$. Следовательно, $\mu_{S} \leqslant p-1$. Тогда из определения показателя сходимости [4; с. 19] вытекает следующая оценка:

$$
\sum_{k \in \mathbb{N}} \frac{1}{\left|s_{k}\right|^{p-1+\varepsilon}}<\infty \quad \forall \varepsilon>0 .
$$

Из (18) вытекают оценки

$$
\sum_{\operatorname{Im} s_{k}<0} \exp \left(\varepsilon_{1} \operatorname{Im} s_{k}\right)<\infty, \quad \sum_{\operatorname{Im} s_{k} \geqslant 0} \exp \left(-\varepsilon_{1} \operatorname{Im} s_{k}\right)<\infty \quad \forall \varepsilon_{1}>0 .
$$

Следовательно, бесконечные произведения в (17) сходятся абсолютно и равномерно в $\mathbb{C}$. Таким образом, $Q(\lambda) \in P(\mathbb{C})$. Из последних неравенств следует также, что бесконечные произведения

$$
g_{1}(\xi)=\prod_{\operatorname{Im} s_{k} \geqslant 0}\left(1-\xi \exp \left(i s_{k}\right)\right), \quad g_{2}(\xi)=\prod_{\operatorname{Im} s_{k}<0}\left(1-\xi \exp \left(-i s_{k}\right)\right)
$$

являются целыми функции нулевого порядка. Их нулевыми множествами являются соответственно $S_{1}=\left\{\exp \left(-i s_{k}\right), k \in \mathbb{N}\right\}, S_{2}=\left\{\exp \left(i s_{k}\right), k \in \mathbb{N}\right\}$. Согласно [15; теорема 3]

$$
\tau_{g_{1}}(p) \leqslant \varlimsup_{r \rightarrow \infty} \frac{n_{S_{1}}(r)}{p(\ln r)^{p-1}}, \quad \tau_{g_{2}}(p) \leqslant \varlimsup_{r \rightarrow \infty} \frac{n_{S_{2}}(r)}{p(\ln r)^{p-1}} .
$$


Пусть $r>1$. Заметим, что выполняются следующие оценки:

$$
n_{S_{1}}(r) \leqslant n_{S}(R), \quad n_{S_{2}}(r) \leqslant n_{S}(R),
$$

где $R=\sqrt{(\ln r)^{2}+4 \pi^{2}}$. Заметим, что $(R / \pi) \cdot n_{S}(R) \leqslant n_{S_{G}}(R \sqrt{2})$. Так как $G(\lambda)$ имеет тип $<q$ при порядке $p$, то согласно [4; гл. 4] выполняется неравенство

$$
\varlimsup_{R \rightarrow \infty} \frac{n_{S_{G}}(R)}{R^{p}}<e p q
$$

Следовательно,

$$
\varlimsup_{R \rightarrow \infty} \frac{n_{S}(R)}{R^{p-1}}<e p(\sqrt{2})^{p} q
$$

Из (19) получаем, что

$$
\frac{n_{S_{2}}(r)}{(\ln r)^{p-1}}<\frac{n_{S}(R)}{(\ln r)^{p-1}}=\frac{n_{S}(R)}{\left(R \sqrt{1-4 \pi^{2} / R^{2}}\right)^{p-1}} .
$$

Заметим, что при $R>2 \sqrt{2} \pi$ выполняется $\sqrt{1-4 \pi^{2} / R^{2}}>1 / \sqrt{2}$. Следовательно,

$$
\varlimsup_{r \rightarrow \infty} \frac{n_{S_{2}}(r)}{(\ln r)^{p-1}}<\varlimsup_{r \rightarrow \infty} \frac{n_{S}(R)}{R^{p-1}}(\sqrt{2})^{p-1} .
$$

Отсюда и из (20) следует, что

$$
\varlimsup_{r \rightarrow \infty} \frac{n_{S_{2}}(r)}{(\ln r)^{p-1}}<e p(\sqrt{2})^{2 p-1} q \pi .
$$

Аналогично доказывается, что

$$
\varlimsup_{r \rightarrow \infty} \frac{n_{S_{1}}(r)}{(\ln r)^{p-1}}<e p(\sqrt{2})^{2 d-1} q \pi .
$$

Следовательно,

$$
\tau_{g_{1}}(p)<e(\sqrt{2})^{2 p-1} q \pi=\delta, \quad \tau_{g_{2}}(p)<e(\sqrt{2})^{2 p-1} q \pi=\delta .
$$

Составим функцию $g(\xi)=g_{1}(1 / \xi) g_{2}(\xi)$. Очевидно, $g_{1}(1 / \xi)$ ограничена при $|\xi| \rightarrow \infty$, а $g_{2}(\xi)$ ограничена при $|\xi| \rightarrow 0$. Заметим, что при замене переменной $\xi=\exp (i \lambda)$ функция $g(\xi)$ перейдет в функцию $Q(\lambda)$. Таким образом, получаем

$$
\varlimsup_{r \rightarrow \infty} \frac{\ln \max _{|\operatorname{Im} \lambda|=r}|Q(\lambda)|}{r^{p}}=\varlimsup_{r \rightarrow \infty} \frac{\ln \max _{|\ln | \xi||=r}|g(\xi)|}{r^{p}}<\delta .
$$

Отсюда следует утверждение леммы.

Обозначим $\xi_{k}=e^{i \lambda_{k}}, \xi_{k}^{\prime}=e^{i \lambda_{k}^{\prime}}, \xi_{k}^{\prime \prime}=e^{i \lambda_{k}^{\prime \prime}}, k \in \mathbb{N}$. Рассмотрим функцию $\hat{b}(\lambda)$, определенную во введении. Так как $\widehat{B}(\lambda) \in E\left[\rho^{*}, \sigma^{*}\right)$, то $\mu_{\Lambda} \leqslant \rho^{*}$. Тогда, повторив рассуждения из доказательства леммы 3 , заключаем, что $\hat{b}(\lambda) \in P(\mathbb{C})$, а бесконечные произведения $f_{1}(\xi)=\prod_{k}\left(1-\xi \xi_{k}^{\prime}\right), f_{2}(\xi)=\prod_{k}\left(1-\xi / \xi_{k}^{\prime \prime}\right), \xi \in \mathbb{C}$, являются целыми функциями. Их нулевыми множествами являются соответственно $\Omega_{1}, \Omega_{2}$. Будем 
теперь считать, что множество $\Lambda$ удовлетворяет условиям теоремы 1 . Тогда из (16) следует, что $\tau_{f_{1}}\left(\rho^{*}\right)=\tau_{1}<\sigma^{*}, \tau_{f_{2}}\left(\rho^{*}\right)=\tau_{2}<\sigma^{*}$. Обозначим $\breve{f}_{1}(\xi)=f_{1}(1 / \xi)$ и составим функцию $\tilde{b}(\xi)=\breve{f}_{1}(\xi) f_{2}(\xi)$. Нетрудно видеть, что $\tilde{b}(\xi) \in H\left[\rho^{*}, \sigma^{*}\right)$. При замене переменной $\xi=e^{i \lambda}$ функция $\tilde{b}(\xi)$ перейдет в функцию $\hat{b}(\lambda)$. Таким образом, $\hat{b}(\lambda) \in P\left[\rho^{*}, \sigma^{*}\right)$. Заметим, что отсюда следует, что $\mu_{\Lambda} \leqslant \rho^{*}-1$. Таким образом, если множество $\Lambda$ удовлетворяет условиям теоремы 1 , то $\mu_{\Lambda} \leqslant \rho^{*}-1$.

Так как $\hat{b}(\lambda) \in P\left[\rho^{*}, \sigma^{*}\right)$, по теореме 2 найдется единственный функционал $b \in$ $A^{\prime}[\rho, \sigma]$ такой, что $\hat{b}(\lambda)=\sum_{n \in \mathbb{Z}} b(n) \exp (i n \lambda)$, т.е. функция $\hat{b}(\lambda)$ является преобразованием Фурье-Лапласа функционала $b$. Очевидно, функция $\tilde{b}(\xi)$ является преобразованием Меллина функционала $b$ и имеет вид $\tilde{b}(\xi)=\sum_{n \in \mathbb{Z}} b(n) \xi^{n}$.

Теорема 4. Пусть $g(\xi) \in H\left[\rho^{*}, \sigma^{*}\right),\left\{z_{k}\right\}_{k \in \mathbb{Z}}-$ нулевое множество $g(\xi)$ (каждый нуль выписъвается столъко раз, какова его кратность). Тогда

$$
g(\xi)=C \xi^{t} g_{1}\left(\frac{1}{\xi}\right) g_{2}(\xi), \quad \text { əдe } \quad g_{1}(\xi)=\prod_{\left|z_{k}\right| \leqslant 1}\left(1-z_{k} \xi\right), \quad g_{2}(\xi)=\prod_{\left|z_{k}\right|>1}\left(1-\frac{\xi}{z_{k}}\right)
$$

$C \in \mathbb{C}, t \in \mathbb{Z}-$ некоторье константь. При этом $g_{1}, g_{2} \in H(\mathbb{C}), \tau_{g_{1}}\left(\rho^{*}\right)<\sigma^{*}$, $\tau_{g_{2}}\left(\rho^{*}\right)<\sigma^{*}$.

ДокАзАтЕЛьство. При замене переменной $\xi=\exp (i \lambda)$ функция $g(\xi)$ перейдет в функцию $L(\lambda) \in P\left[\rho^{*}, \sigma^{*}\right)$. Тогда из леммы 3 следует, что функция

$$
Q(\lambda)=\prod_{\left|z_{k}\right| \leqslant 1}\left(1-\frac{z_{k}}{\exp (i \lambda)}\right) \prod_{\left|z_{k}\right|>1}\left(1-\frac{\exp (i \lambda)}{z_{k}}\right)
$$

принадлежит $P(\mathbb{C})$ и имеет порядок не выше $\rho^{*}$. Введем функцию $w(\lambda)=L(\lambda) / Q(\lambda)$. Очевидно, $w(\lambda)$ принадлежит $P(\mathbb{C})$ и не имеет нулей. Так как $L(\lambda)$ и $Q(\lambda)$ имеют порядки не выше $\rho^{*}$, то [4; c. 37] $w(\lambda)$ имеет порядок не выше $\rho^{*}$. Так как $w(\lambda)$ нигде не обращается в нуль и имеет порядок не выше $\rho^{*}$, то $[14 ;$ c. 272,282$] w(\lambda)=e^{p(\lambda)}$, где $p(\lambda)$ является многочленом степени не выше, чем целая часть $\rho^{*}$. Так как $w(\lambda)$ имеет период $2 \pi$, многочлен $p(\lambda)$ должен удовлетворять условию

$$
p(\lambda+2 \pi)-p(\lambda)=i t 2 \pi \quad \forall \lambda \in \mathbb{C}, \quad \exists t \in \mathbb{Z} .
$$

Нетрудно видеть, что условию (21) удовлетворяют только многочлены вида $p(\lambda)=$ $i t \lambda+C_{0}$, где $t \in \mathbb{Z}, C_{0} \in \mathbb{C}$ - произвольные константы. Таким образом, $w(\lambda)=C e^{i t \lambda}$, где $C=e^{C_{0}}$. Следовательно, $L(\lambda)=C \exp (i t \lambda) Q(\lambda)$. Отсюда следует, что $g(\xi)=$ $C \xi^{t} g_{1}(1 / \xi) g_{2}(\xi)$.

Осталось оценить $\tau_{g_{1}}\left(\rho^{*}\right), \tau_{g_{2}}\left(\rho^{*}\right)$. Из теоремы об оценке снизу аналитической функции, не обращающейся в нуль [4; с. 31], следует, что найдутся некоторые положительные константы $C_{1}, C_{2}$, при которых выполняются оценки

$$
\left|g_{1}\left(\frac{1}{\xi}\right)\right| \geqslant C_{1}, \quad 1<|\xi|<\infty, \quad\left|g_{2}(\xi)\right| \geqslant C_{2}, \quad 0<|\xi|<1 .
$$


Так как $g \in H\left[\rho^{*}, \sigma^{*}\right)$, найдутся числа $C_{3}>0, j \in \mathbb{N}$ такие, что в $\mathbb{C} \backslash\{0\}$ выполняется оценка $|g(\xi)| \leqslant C_{3} \exp \left(\sigma_{j}^{*}|\ln | \xi \mid \rho^{*}\right)$. Тогда

$$
\begin{array}{cc}
\left|g_{2}(\xi)\right| \leqslant \frac{C_{3} \exp \left(\sigma_{j}^{*}|\ln | \xi||^{\rho^{*}}\right)}{C \xi^{t} g_{1}(1 / \xi)} \leqslant \frac{C_{3} \exp \left(\sigma_{j}^{*}|\ln | \xi||^{\rho^{*}}\right)}{C \xi^{t} C_{1}}, \quad 1<|\xi|<\infty, \\
\left|g_{1}\left(\frac{1}{\xi}\right)\right| \leqslant \frac{C_{3} \exp \left(\sigma_{j}^{*}|\ln | \xi||^{\rho^{*}}\right)}{C \xi^{t} g_{2}(\xi)} \leqslant \frac{C_{3} \exp \left(\sigma_{j}^{*}|\ln | \xi||^{\rho^{*}}\right)}{C \xi^{t} C_{2}}, \quad 0<|\xi|<1 .
\end{array}
$$

Последнее неравенство можно переписать в виде

$$
\left|g_{1}(\xi)\right| \leqslant \frac{C_{3} \xi^{t} \exp \left(\sigma_{j}^{*}|\ln | \xi||^{\rho^{*}}\right)}{C C_{2}}, \quad 1<|\xi|<\infty .
$$

Отсюда получаем

$$
\begin{array}{ll}
\ln \left|g_{2}(\xi)\right| \leqslant \sigma_{j}^{*}|\ln | \xi||^{\rho^{*}}\left(1-\frac{\xi^{t}}{\sigma_{j}^{*}|\ln | \xi \mid \rho^{*}}\right)+\text { const, } & 1<|\xi|<\infty \\
\ln \left|g_{1}(\xi)\right| \leqslant \sigma_{j}^{*}|\ln | \xi||^{\rho^{*}}\left(1+\frac{\xi^{t}}{\sigma_{j}^{*}|\ln | \xi|| \rho^{*}}\right)+\text { const, } & 1<|\xi|<\infty
\end{array}
$$

Из последних соотношений следуют оценки $\tau_{g_{1}}\left(\rho^{*}\right)<\sigma^{*}, \tau_{g_{2}}\left(\rho^{*}\right)<\sigma^{*}$.

5. Дискретное уравнение свертки. Пусть $m \in \mathbb{Z}$. Определим на $A[\rho, \sigma]$ оператор сдвига $S^{m}$, который каждой функции $\psi \in A[\rho, \sigma]$ ставит в соответствие функцию $S^{m} \psi \in A$ такую, что $S^{m} \psi(n) \equiv \psi(n+m)$. Нетрудно проверить, что $S^{m}$ действует линейно и непрерывно из $A[\rho, \sigma]$ в $A[\rho, \sigma]$. Пусть $\varphi \in A^{\prime}[\rho, \sigma]$. Определим на $A[\rho, \sigma]$ оператор свертки $M_{\varphi}$, который каждой функции $\psi \in A[\rho, \sigma]$ ставит в соответствие функцию $M_{\varphi}[\psi] \in A$ такую, что $M_{\varphi}[\psi](m) \equiv\left\langle\varphi, S^{m} \psi\right\rangle$. Нетрудно установить, что оператор $M_{\varphi}$ действует линейно и непрерывно из пространства $A[\rho, \sigma]$ в пространство $A[\rho, \tilde{\sigma}]$, где число $\tilde{\sigma}>\sigma$ зависит от $\varphi$.

Как уже было отмечено, функция $\hat{b}(\lambda) \in P\left[\rho^{*}, \sigma^{*}\right)$ является преобразованием Фурье-Лапласа функционала $b \in A^{\prime}[\rho, \sigma]$. Функционал $b$ определяет на $A[\rho, \sigma]$ однородное уравнение свертки $M_{b}[\psi](m)=0 \quad \forall m \in \mathbb{Z}$, совпадающее с уравнением (6). Обозначим через $W_{b}$ пространство решений уравнения (6). Так как оператор $M_{b}$ является линейным и непрерывным, то $W_{b}=\operatorname{ker} M_{b}$ является замкнутым подпространством в $A[\rho, \sigma]$. Функция $\hat{b}(\lambda)$ называется характеристической функцией уравнения (6). Обозначим нулевое множество $\tilde{b}(\xi)$ через $\widetilde{\Lambda}$. Множество $\widetilde{\Lambda}$ состоит из точек $\xi_{k}, k \in \mathbb{N}$. Очевидно, функции $x_{k}(n)=\exp \left(i n \lambda_{k}\right)=\xi_{k}^{n}, k \in \mathbb{N}$, являются решениями уравнения (6). Эти решения будем называть элементарными.

6. Теорема деления в $H\left[\rho^{*}, \sigma^{*}\right)$.

Tеорема 5. Пусть функиии $h_{1}(\xi), h_{2}(\xi) \in H\left[\rho^{*}, \sigma^{*}\right)$ maковы, что $\left(h_{1} / h_{2}\right)(\xi) \in$ $H_{0}$. Тогда $\left(h_{1} / h_{2}\right)(\xi) \in H\left[\rho^{*}, \sigma^{*}\right)$.

Доказательство. Пусть $Z^{\prime}=\left\{z_{k}^{\prime}\right\}_{k \in \mathbb{Z}}-$ нулевое множество $h_{1}(\xi), Z^{\prime \prime}=\left\{z_{k}^{\prime \prime}\right\}_{k \in \mathbb{Z}}$ - нулевое множество $h_{2}(\xi)$ (нули повторяются столько раз, какова их кратность). 
Так как $\left(h_{1} / h_{2}\right)(\xi) \in H_{0}$, то $Z^{\prime \prime} \subset Z^{\prime}$. Обозначим

$$
\begin{array}{ll}
h_{11}(\xi)=\prod_{\left|z_{k}^{\prime}\right| \leqslant 1}\left(1-z_{k}^{\prime} \xi\right), & h_{12}(\xi)=\prod_{\left|z_{k}^{\prime}\right|>1}\left(1-\frac{\xi}{z_{k}^{\prime}}\right), \\
h_{21}(\xi)=\prod_{\left|z_{k}^{\prime \prime}\right| \leqslant 1}\left(1-z_{k}^{\prime \prime} \xi\right), & h_{22}(\xi)=\prod_{\left|z_{k}^{\prime \prime}\right|>1}\left(1-\frac{\xi}{z_{k}^{\prime \prime}}\right) .
\end{array}
$$

Тогда согласно теореме 4

$$
h_{1}(\xi)=C_{1} \xi^{t_{1}} h_{11}\left(\frac{1}{\xi}\right) h_{12}(\xi), \quad h_{2}(\xi)=C_{2} \xi^{t_{2}} h_{21}\left(\frac{1}{\xi}\right) h_{22}(\xi),
$$

где $C_{1}, C_{2} \in \mathbb{C}, t_{1}, t_{2} \in \mathbb{Z}$ - некоторые константы. При этом

$$
\tau_{h_{11}}\left(\rho^{*}\right)<\sigma^{*}, \quad \tau_{h_{12}}\left(\rho^{*}\right)<\sigma^{*}, \quad \tau_{h_{21}}\left(\rho^{*}\right)<\sigma^{*}, \quad \tau_{h_{22}}\left(\rho^{*}\right)<\sigma^{*} .
$$

Рассмотрим функции $w_{1}(\xi)=h_{11}(\xi) / h_{21}(\xi), w_{2}(\xi)=h_{12}(\xi) / h_{22}(\xi)$. Тогда

$$
\frac{h_{1}(\xi)}{h_{2}(\xi)}=C \xi^{t} w_{1}\left(\frac{1}{\xi}\right) w_{2}(\xi)
$$

где $C=C_{1} / C_{2}, t=t_{1}-t_{2}$. Очевидно, $w_{1}(\xi), w_{2}(\xi)$ являются целыми функциями. Кроме того, из (16) следует, что

$$
\tau_{w_{1}}\left(\rho^{*}\right) \leqslant \tau_{h_{11}}\left(\rho^{*}\right)<\sigma^{*}, \quad \tau_{w_{2}}\left(\rho^{*}\right) \leqslant \tau_{h_{12}}\left(\rho^{*}\right)<\sigma^{*} .
$$

Отсюда вытекает, что $\left(h_{1} / h_{2}\right)(\xi) \in H\left[\rho^{*}, \sigma^{*}\right)$.

7. Аппроксимация решений. Пусть даны функции $g_{1}(\xi), g_{2}(\xi) \in H\left[\rho^{*}, \sigma^{*}\right)$ такие, что $\left(g_{1} \cdot g_{2}\right)(\xi) \in H\left[\rho^{*}, \sigma^{*}\right)$. Обозначим $g_{3}(\xi)=\left(g_{1} \cdot g_{2}\right)(\xi)$. По теореме 3 найдутся функционалы $\varphi_{1}, \varphi_{2}, \varphi_{3} \in A^{\prime}[\rho, \sigma]$ такие, что $\widetilde{\varphi}_{1}(\xi) \equiv g_{1}, \widetilde{\varphi}_{2}(\xi) \equiv g_{2}(\xi)$, $\widetilde{\varphi}_{3}(\xi) \equiv g_{3}(\xi)$. Тогда

$$
\begin{aligned}
\widetilde{\varphi}_{3}(\xi) & =\left\langle\varphi_{3}, \xi^{n}\right\rangle=\widetilde{\varphi}_{1}(\xi) \cdot \widetilde{\varphi}_{2}(\xi)=\sum_{m \in \mathbb{Z}} \varphi_{1}(m) \xi^{m} \sum_{n \in \mathbb{Z}} \varphi_{2}(n) \xi^{n} \\
& =\sum_{m \in \mathbb{Z}} \varphi_{1}(m) \sum_{n \in \mathbb{Z}} \varphi_{2}(n) \xi^{n+m}=\left\langle\varphi_{1}, M_{\varphi_{2}}\left[\xi^{n}\right]\right\rangle .
\end{aligned}
$$

Таким образом, функционал $\varphi_{3}$ действует по правилу $\left\langle\varphi_{3}, \cdot\right\rangle=\left\langle\varphi_{1}, M_{\varphi_{2}}[\cdot]\right\rangle$. Функционал $\varphi_{3}$, действующий по этому правилу, называется сверткой функционалов $\varphi_{1}$ и $\varphi_{2}$ и обозначается $\varphi_{3}=\varphi_{1} * \varphi_{2}$.

Теорема 6. Имеем $W_{b}=\overline{\operatorname{span}\left\{x_{k}, k \in \mathbb{N}\right\}}$.

ДокАЗАТЕЛЬСтво. Пусть $\varphi \in A^{\prime}[\rho, \sigma]$ - произвольный функционал такой, что

$$
\left\langle\varphi, x_{k}\right\rangle=0 \quad \forall k \in \mathbb{N} .
$$

Согласно принципу аппроксимации [16; теорема 2.3.1] для доказательства теоремы достаточно показать, что $\langle\varphi, \psi\rangle=0 \quad \forall \psi \in W_{b}$. Рассмотрим $\widetilde{\varphi}(\xi)=\sum_{n \in \mathbb{Z}} \varphi(n) \xi^{n}$. 
По теореме $3 \widetilde{\varphi}(\xi) \in H\left[\rho^{*}, \sigma^{*}\right)$. Определим функцию $\alpha(\xi)=\widetilde{\varphi}(\xi) / \tilde{b}(\xi)$. В силу условия $(23) \alpha(\xi) \in H_{0}$. Тогда по теореме $5 \alpha(\xi) \in H\left[\rho^{*}, \sigma^{*}\right)$. Следовательно, по теореме 3 найдется функционал $\chi \in A^{\prime}[\rho, \sigma]$ такой, что $\widetilde{\chi}(\xi) \equiv \alpha(\xi)$. Так как $\widetilde{\varphi}(\xi)=$ $\tilde{\chi}(\xi) \cdot \tilde{b}(\xi)$, то $\varphi=\chi * b$. Следовательно,

$$
\langle\varphi, \psi\rangle=\langle\chi * b, \psi\rangle=\left\langle\chi, M_{b}[\psi]\right\rangle=\langle\chi, 0\rangle=0 \quad \forall \psi \in W_{b} .
$$

8. Базис в пространстве $W_{b}$. Выясним, при каких условиях на множество $\Lambda$ выполняется утверждение

(A) функции $x_{k}, k \in \mathbb{N}$, образуют регулярный базис в пространстве $W_{b}$. Рассмотрим следующие условия:

(Q1) для любой последовательности $\left\{t_{k}\right\}_{k \in \mathbb{N}} \subset \mathbb{C}$, удовлетворяющей условию

$$
\exists j \in \mathbb{N}: \quad \sup _{k \in \mathbb{N}} \frac{\left|t_{k}\right|}{\left\|e^{i n \lambda_{k}}\right\|_{A_{j}}}<\infty,
$$

найдется функционал $\varphi \in A^{\prime}[\rho, \sigma]$ такой, что $\left\langle\varphi, e^{i n \lambda_{k}}\right\rangle=t_{k} \forall k \in \mathbb{N}$;

(Q2) для любой последовательности $\left\{t_{k}\right\}_{k \in \mathbb{N}} \subset \mathbb{C}$, удовлетворяющей условию

$$
\exists j \in \mathbb{N}: \quad \sup _{k \in \mathbb{N}} \frac{\left|t_{k}\right|}{\exp \left(\sigma_{j}^{*}\left|\operatorname{Im} \lambda_{k}\right|^{\rho^{*}}\right)}<\infty,
$$

найдется функция $g \in P\left[\rho^{*}, \sigma^{*}\right)$ такая, что $g\left(\lambda_{k}\right)=t_{k} \forall k$;

(Q3) для любой последовательности $\left\{t_{k}\right\}_{k \in \mathbb{N}} \subset \mathbb{C}$, удовлетворяющей условию

$$
\exists j \in \mathbb{N}: \quad \sup _{k \in \mathbb{N}} \frac{\left|t_{k}\right|}{\exp \left(\sigma_{j}^{*}|\ln | \xi_{k}||^{*}\right)}<\infty
$$

найдется функция $\omega \in H\left[\rho^{*}, \sigma^{*}\right)$ такая, что $\omega\left(\xi_{k}\right)=t_{k} \quad \forall k$.

$$
\text { Лемма 4. Имеем (Q3) }
$$

ДоказАтельство. 1) Соотношение $(\mathbf{Q 1}) \Longleftrightarrow(\mathbf{A})$ следует из [11; теорема 1], с учетом того, что $W_{b}=\overline{\operatorname{span}\left\{e^{i n \lambda_{k}}, k \in \mathbb{N}\right\}}$ (последнее было доказано в теореме 6 ).

2) Соотношение $(\mathbf{Q 3}) \Longleftrightarrow(\mathbf{Q 2})$ очевидно. Действительно, условия (25) и (26) суть одно и тоже; функции $\omega(\xi)$ и $g(\lambda)$ получаются друг из друга при замене переменной $\xi=\exp (i \lambda)$.

3) Докажем теперь, что $(\mathbf{Q 2}) \Longleftrightarrow(\mathbf{Q 1})$. По теореме 2 существование функции $g(\lambda) \in P\left[\rho^{*}, \sigma^{*}\right)$ такой, что $g\left(\lambda_{k}\right)=t_{k} \quad \forall k \in \mathbb{N}$, равносильно существованию функционала $\varphi \in A^{\prime}[\rho, \sigma]$ такого, что $\left\langle\varphi, e^{i n \lambda_{k}}\right\rangle=t_{k} \forall k \in \mathbb{N}$. Осталось доказать равносильность условий (25) и (24). Итак, пусть выполнено (25). Тогда с учетом (12) получаем

$$
\sup _{k \in \mathbb{N}} \frac{\left|t_{k}\right|}{\left\|e^{i n \lambda_{k}}\right\|_{A_{j+1}}} \leqslant \text { const } \cdot \sup _{k \in \mathbb{N}} \frac{\left|t_{k}\right|}{\exp \left(\sigma_{j}^{*}\left|\operatorname{Im} \lambda_{k}\right|^{\rho^{*}}\right)}<\infty,
$$

т.е. выполняется (24). Наоборот, пусть выполнено (24). Тогда с учетом (11) получаem

$$
\frac{\left|t_{k}\right|}{\exp \left(\sigma_{j}^{*}\left|\operatorname{Im} \lambda_{k}\right|^{\rho^{*}}\right)}<\sup _{k \in \mathbb{N}} \frac{\left|t_{k}\right|}{\left\|e^{i n \lambda_{k}}\right\|_{A_{j}}}<\infty,
$$

т.е. выполняется (25). 
Рассмотрим теперь условие

(Q3a) для любых последовательностей $\left\{t_{k}^{\prime}\right\}_{k \in \mathbb{N}} \subset \mathbb{C}$ и $\left\{t_{k}^{\prime \prime}\right\}_{k \in \mathbb{N}} \subset \mathbb{C}$, удовлетворяющих условию

$$
\exists j \in \mathbb{N}: \quad \sup _{k \in \mathbb{N}} \frac{\left|t_{k}^{\prime}\right|}{\exp \left(\sigma_{j}^{*}|\ln | \xi_{k}^{\prime} \mid \rho^{*}\right)}<\infty, \quad \frac{\left|t_{k}^{\prime \prime}\right|}{\exp \left(\sigma_{j}^{*}|\ln | \xi_{k}^{\prime \prime} \mid \rho^{*}\right)}<\infty,
$$

найдется функция $\omega(\xi) \in H\left[\rho^{*}, \sigma^{*}\right)$ такая, что $\omega\left(\xi_{k}^{\prime}\right)=t_{k}^{\prime}, \omega\left(\xi_{k}^{\prime \prime}\right)=t_{k}^{\prime \prime} \forall k$.

Очевидно, (Q3a) $\Longleftrightarrow(\mathbf{Q 3})$. Выясним, при каких условиях выполняется (Q3a). Возьмем произвольные последовательности $\left\{t_{k}^{\prime}\right\}_{k \in \mathbb{N}}$ и $\left\{t_{k}^{\prime \prime}\right\}_{k \in \mathbb{N}}$, удовлетворяющие (27). Положим $v_{k}^{\prime}=t_{k}^{\prime} / f_{2}\left(\xi_{k}^{\prime}\right), v_{k}^{\prime \prime}=t_{k}^{\prime \prime} / \breve{f}_{1}\left(\xi_{k}^{\prime \prime}\right)$. Согласно (22) выполняются равномерные по $k$ оценки: $\left|v_{k}^{\prime}\right| \leqslant C_{2}\left|t_{k}^{\prime}\right|,\left|v_{k}^{\prime \prime}\right| \leqslant C_{1}\left|t_{k}^{\prime \prime}\right|$. Следовательно, последовательности $\left\{v_{k}^{\prime}\right\}_{k \in \mathbb{N}}$ и $\left\{v_{k}^{\prime \prime}\right\}_{k \in \mathbb{N}}$ удовлетворяют условиям (27). Предположим, что выполняется условие

$$
\lim _{k \rightarrow \infty} \frac{1}{|\ln | \xi_{k}^{\prime} \mid \rho^{\rho^{*}}} \ln \left|f_{1}^{\prime}\left(\frac{1}{\xi_{k}^{\prime}}\right) \cdot \frac{1}{\xi_{k}^{\prime}}\right|=\tau_{1} .
$$

Тогда согласно [17; теорема 10] существует $\gamma(\xi) \in H(\mathbb{C})$ такая, что $\tau_{\gamma}\left(\rho^{*}\right)<\sigma^{*}$, $\gamma\left(1 / \xi_{k}^{\prime}\right)=v_{k}^{\prime} \quad \forall k$. Положим $\omega_{1}(\xi)=\gamma(1 / \xi)$. Тогда $\omega_{1}\left(\xi_{k}^{\prime}\right)=v_{k}^{\prime}$. Аналогично, если выполняется

$$
\lim _{k \rightarrow \infty} \frac{1}{|\ln | \xi_{k}^{\prime \prime}||^{\rho^{*}}} \ln \left|f_{2}^{\prime}\left(\xi_{k}^{\prime \prime}\right) \cdot \xi_{k}^{\prime \prime}\right|=\tau_{2}
$$

то существует целая функция $\omega_{2}(\xi)$ такая, что $\tau_{\omega_{2}}\left(\rho^{*}\right)<\sigma^{*}, \omega_{2}\left(\xi_{k}^{\prime \prime}\right)=v_{k}^{\prime \prime} \forall k$. Определим функцию $\omega(\xi)=\omega_{1}(\xi) f_{2}(\xi)+\omega_{2}(\xi) \breve{f}_{1}(\xi)$. Очевидно, $\omega_{1}(\xi) \cdot f_{2}(\xi) \in H_{0}$, $\omega_{2}(\xi) \cdot \breve{f}_{1}(\xi) \in H_{0}$. Заметим, что функции $\breve{f}_{1}(\xi), \omega_{1}(\xi)$ ограничены при $|\xi| \rightarrow \infty$, а функции $f_{2}(\xi), \omega_{2}(\xi)$ ограничены при $|\xi| \rightarrow 0$. Так как $\tau_{\omega_{2}}\left(\rho^{*}\right)<\sigma^{*}, \tau_{\gamma}\left(\rho^{*}\right)<\sigma^{*}$, $\tau_{f_{2}}\left(\rho^{*}\right)<\sigma^{*}, \tau_{f_{1}}\left(\rho^{*}\right)<\sigma^{*}$, то $\omega_{1}(\xi) \cdot f_{2}(\xi) \in H\left[\rho^{*}, \sigma^{*}\right), \omega_{2}(\xi) \cdot \breve{f}_{1}(\xi) \in H\left[\rho^{*}, \sigma^{*}\right)$. Следовательно, $\omega(\xi) \in H\left[\rho^{*}, \sigma^{*}\right)$. Кроме того, $\omega\left(\xi_{k}^{\prime}\right)=t_{k}^{\prime}, \omega\left(\xi_{k}^{\prime \prime}\right)=t_{k}^{\prime \prime} \forall k$. Действительно,

$$
\begin{aligned}
& \omega\left(\xi_{k}^{\prime}\right)=\omega_{1}\left(\xi_{k}^{\prime}\right) f_{2}\left(\xi_{k}^{\prime}\right)+\omega_{2}\left(\xi_{k}^{\prime}\right) \breve{f}_{1}\left(\xi_{k}^{\prime}\right)=v_{k}^{\prime} f_{2}\left(\xi_{k}^{\prime}\right)+\omega_{2}\left(\xi_{k}^{\prime}\right) \cdot 0=\frac{t_{k}^{\prime}}{f_{2}\left(\xi_{k}^{\prime}\right)} f_{2}\left(\xi_{k}^{\prime}\right)=t_{k}^{\prime}, \\
& \omega\left(\xi_{k}^{\prime \prime}\right)=\omega_{1}\left(\xi_{k}^{\prime \prime}\right) f_{2}\left(\xi_{k}^{\prime \prime}\right)+\omega_{2}\left(\xi_{k}^{\prime \prime}\right) \breve{f}_{1}\left(\xi_{k}^{\prime \prime}\right)=\omega_{1}\left(\xi_{k}^{\prime \prime}\right) \cdot 0+v_{k}^{\prime \prime} \breve{f}_{1}\left(\xi_{k}^{\prime \prime}\right)=\frac{t_{k}^{\prime \prime}}{\breve{f}_{1}\left(\xi_{k}^{\prime \prime}\right)} \breve{f}_{1}\left(\xi_{k}^{\prime \prime}\right)=t_{k}^{\prime \prime} .
\end{aligned}
$$

Таким образом, при выполнении (28) и (29) выполняется (Q3a), а значит, и (A). Нетрудно видеть, что условия (28), (29) равносильны соответственно условиям

$$
\begin{aligned}
& \lim _{k \rightarrow \infty} \frac{1}{|\ln | \xi_{k}^{\prime} \mid \rho^{*}} \ln \left|\prod_{n \neq k}\left(1-\frac{\xi_{n}^{\prime}}{\xi_{k}^{\prime}}\right)\right|=\tau_{1}, \\
& \lim _{k \rightarrow \infty} \frac{1}{|\ln | \xi_{k}^{\prime \prime} \mid \rho^{*}} \ln \left|\prod_{n \neq k}\left(1-\frac{\xi_{k}^{\prime \prime}}{\xi_{n}^{\prime \prime}}\right)\right|=\tau_{2} .
\end{aligned}
$$

Таким образом, доказано следующее утверждение.

Теорема 7. Если множество $\widetilde{\Lambda}$ удовлетворяет условиям (30) и (31), то функиии $x_{k}, k \in \mathbb{N}$, образуют регулярный базис в пространстве $W_{b}$. 
Заметим, что условия (30), (31) равносильны соответственно условиям (3), (4). Таким образом, получаем следующее утверждение.

Теорема 8. Если множество $\Lambda$ удовлетворяет условиям теоремы 1, то функиии $x_{k}, k \in \mathbb{N}$, образуют регулярный базис в $W_{b}$.

9. Формулы для коэффициентов. Пусть $a \in W_{b}$. Если выполнены условия теоремы 1 , то по теореме 8 функция $а$ единственным образом разлагается в ряд $a(n)=\sum_{k=1}^{\infty} c_{k} \exp \left(i n \lambda_{k}\right)$. Очевидно, функции

$$
\hat{b}_{k}(\lambda)=\frac{\hat{b}(\lambda)}{1-\exp \left(i \lambda-i \lambda_{k}\right)}, \quad k \in \mathbb{N},
$$

принадлежат $P\left[\rho^{*}, \sigma^{*}\right)$ и разлагаются в ряды Фурье $\hat{b}_{k}(\lambda)=\sum_{n \in \mathbb{Z}} b_{k}(n) \exp ($ in $\lambda)$. Следовательно, $b_{k} \in A^{\prime}[\rho, \sigma], k \in \mathbb{N}$. Тогда

$$
\left\langle b_{m}, a\right\rangle=\left\langle b_{m}, \sum_{k=1}^{\infty} c_{k} \exp \left(i n \lambda_{k}\right)\right\rangle=\sum_{k=1}^{\infty} c_{k}\left\langle b_{m}, \exp \left(i n \lambda_{k}\right)\right\rangle=c_{m} \widehat{b}_{m}\left(\lambda_{m}\right), \quad m \in \mathbb{N} .
$$

Таким образом,

$$
c_{m}=\frac{\left\langle b_{m}, a\right\rangle}{\hat{b}_{m}\left(\lambda_{m}\right)}=\frac{\sum_{n \in \mathbb{Z}} b_{m}(n) a(n)}{\hat{b}_{m}\left(\lambda_{m}\right)}, \quad m \in \mathbb{N} .
$$

10. Базис в пространстве $W_{B}$. Как было отмечено ранее, для того, чтобы функции $\exp \left(i z \lambda_{k}\right), k \in \mathbb{N}$, образовывали регулярный базис в $W_{B}$, необходимо и достаточно, чтобы выполнялось (Q).

Лемма 5. Имеем (Q2) $\Longrightarrow(\mathbf{Q})$.

ДокАЗАТЕЛЬСтво. Пусть выполнено (Q2). Покажем, что выполняется (Q). Возьмем произвольную последовательность $\left\{t_{k}\right\}_{k \in \mathbb{N}} \subset \mathbb{C}$, удовлетворяющую условию (8). Заметим, что при $\left|\operatorname{Im} \lambda_{k}\right| \neq 0$ выполняется

$$
\left|\lambda_{k}\right| \leqslant\left|\operatorname{Re} \lambda_{k}\right|+\left|\operatorname{Im} \lambda_{k}\right|<\left|\operatorname{Im} \lambda_{k}\right|+2 \pi=\left|\operatorname{Im} \lambda_{k}\right|\left(1+\frac{2 \pi}{\left|\operatorname{Im} \lambda_{k}\right|}\right) .
$$

Так как

$$
\lim _{k \rightarrow \infty}\left(1+\frac{2 \pi}{\left|\operatorname{Im} \lambda_{k}\right|}\right)=1,
$$

при достаточно больших $k$ выполняется $\sigma_{j}^{*}\left|\lambda_{k}\right|^{\rho^{*}}<\sigma_{j+1}^{*}\left|\operatorname{Im} \lambda_{k}\right|^{\rho^{*}}$. Отсюда следует, что последовательность $\left\{t_{k}\right\}_{k \in \mathbb{N}}$ удовлетворяет условию (25). Так как, по предположению, выполнено (Q2), найдется функция $g(\lambda) \in P\left[\rho^{*}, \sigma^{*}\right)$ такая, что $g\left(\lambda_{k}\right)=t_{k}$ $\forall k$. Так как $g \in P\left[\rho^{*}, \sigma^{*}\right)$, очевидно, $g \in E\left[\rho^{*}, \sigma^{*}\right)$. Следовательно, выполняется (Q).

Из леммы 5 и теоремы 8 вытекает следующее утверждение.

ТЕорема 9. Если множество $\Lambda$ удовлетворяет условиям теоремы 1, то въполняется условие (Q2).

СлЕДСТвИЕ. При выполнении условий теоремы 1 бункции $\exp \left(i \lambda_{k} z\right), k \in \mathbb{N}$, образуют регулярный базис в пространстве $W_{B}$. 
11. Изоморфизм между $W_{b}$ и $W_{B}$. Введем отображение $T: E[\rho, \sigma] \rightarrow A[\rho, \sigma]$, которое каждой функции $f \in E[\rho, \sigma]$ ставит в соответствие функцию $\left.T[f] \equiv f\right|_{\mathbb{Z}} \in$ $A[\rho, \sigma]$.

ТЕОРема 10. Если выполняются условия теоремы 1, то отображение T осуществляет линейный топологический изоморфизм между пространствами $W_{B}$ $u W_{b}$.

Доказательство. 1) Пусть $f \in W_{B}$. По следствию к теореме 9 функцию $f$ можно единственным образом разложить в ряд $f(z)=\sum_{k=1}^{\infty} d_{k} \exp \left(i z \lambda_{k}\right)$. Тогда $T[f](n)=\sum_{k=1}^{\infty} d_{k} \exp \left(i n \lambda_{k}\right)$. Следовательно, $T[f] \in W_{b}$.

2) Пусть $a \in W_{b}$. По теореме 8 функцию $a$ можно единственным образом разложить в ряд $a(n)=\sum_{k=1}^{\infty} c_{k} \exp \left(i n \lambda_{k}\right)$. Рассмотрим ряд

$$
h(z)=\sum_{k=1}^{\infty} c_{k} \exp \left(i z \lambda_{k}\right) .
$$

Докажем сходимость ряда (33) в топологии пространства $E[\rho, \sigma]$. Зафиксируем произвольное $j \in \mathbb{N}$. Так как функции $\exp \left(i \lambda_{k} n\right), k \in \mathbb{N}$, образуют в пространстве $W_{b}$ регулярный базис, то [11; с. 523] выполняется оценка

$$
\sum_{k=1}^{\infty}\left|c_{k}\right| \cdot \| \exp \left(\text { in } \lambda_{k}\right) \|_{A_{j+2}}<\infty .
$$

Из неравенства (12) вытекает оценка

$$
\left\|\exp \left(i n \lambda_{k}\right)\right\|_{A_{j+2}}>\exp \left(\sigma_{j+1}^{*}\left|\operatorname{Im} \lambda_{k}\right|^{\rho^{*}}\right) .
$$

Из (34) и (35) следует

$$
\sum_{k=1}^{\infty}\left|c_{k}\right| \exp \left(\sigma_{j+1}^{*}\left|\operatorname{Im} \lambda_{k}\right|^{\rho^{*}}\right)=: K_{j}<\infty .
$$

Оценим теперь ряд (33):

$$
\begin{aligned}
|h(z)| & \leqslant \sum_{k=1}^{\infty}\left|c_{k}\right|\left|\exp \left(i z \lambda_{k}\right)\right|=\sum_{k=1}^{\infty}\left|c_{k}\right| \exp \left(-\operatorname{Re} z \cdot \operatorname{Im} \lambda_{k}-\operatorname{Im} z \cdot \operatorname{Re} \lambda_{k}\right) \\
& \leqslant \sum_{k=1}^{\infty}\left|c_{k}\right| \exp \left(|z|\left(2 \pi+\left|\operatorname{Im} \lambda_{k}\right|\right)\right) \leqslant \exp \left(\sigma_{j}|z|^{\rho}\right) \sum_{k=1}^{\infty}\left|c_{k}\right| \sup \frac{\exp \left(|z|\left(2 \pi+\left|\operatorname{Im} \lambda_{k}\right|\right)\right)}{\exp \left(\sigma_{j}|z|^{\rho}\right)} \\
& =\exp \left(\sigma_{j}|z|^{\rho}\right) \sum_{k=1}^{\infty}\left|c_{k}\right| \exp \left(\sigma_{j}^{*}\left(2 \pi+\left|\operatorname{Im} \lambda_{k}\right|\right)^{\rho^{*}}\right) .
\end{aligned}
$$

Очевидно, что $\sigma_{j}^{*}\left(2 \pi+\left|\operatorname{Im} \lambda_{k}\right|\right)^{\rho^{*}}<\sigma_{j+1}^{*}\left|\operatorname{Im} \lambda_{k}\right|^{\rho^{*}}, k>k_{1}(j)$. Таким образом, с учетом (36) получаем

$$
|h(z)| \leqslant K_{j} \exp \left(\sigma_{j}|z|^{\rho}\right) \quad \forall z \in \mathbb{C} .
$$


Из (37) следует, что ряд (33) сходится абсолютно и равномерно на любом компакте в $\mathbb{C}$ и, таким образом, $h(z) \in H(\mathbb{C})$. Так как $(37)$ выполняется для произвольного $j \in \mathbb{N}$, то $h(z) \in E[\rho, \sigma]$. Тогда из представления (33) следует, что $h(z) \in W_{B}$. Кроме того, очевидно, $T[h]=a$.

Предположим, что существует функция $h_{1}(z)=\sum_{k=1}^{\infty} c_{k}^{\prime} \exp \left(i z \lambda_{k}\right) \in W_{B}$ такая, что $T\left[h_{1}\right]=a$. Тогда $a(n)=\sum_{k=1}^{\infty} c_{k}^{\prime} \exp \left(i n \lambda_{k}\right)$. Но из единственности разложения по базису $\exp \left(i n \lambda_{k}\right), k \in \mathbb{N}$, в пространстве $W_{b}$ следует, что $c_{k}^{\prime}=c_{k} \forall k$. Следовательно, $h_{1}(z) \equiv h(z)$. Таким образом, $T$ осуществляет взаимно-однозначное отображение между $W_{B}$ и $W_{b}$. Очевидно, отображение $T$ является линейным и непрерывным. Так как $W_{b}$ и $W_{B}$ являются замкнутыми подпространствами пространств Фреше, то $W_{b}$ и $W_{B}$ являются пространствами Фреше. Следовательно [18; с. 175], отображение $T^{-1}$ является линейным и непрерывным.

12. Доказательство основного результата. Пусть выполнены все условия теоремы 1. Тогда согласно теореме 10 любое решение уравнения (1) однозначно определяется своими значениями на $\mathbb{Z}$. Докажем формулу (5). Пусть $f \in W_{B}$. Так как $\left.f\right|_{\mathbb{Z}} \in W_{b}$, то $f(n)=\sum_{k=1}^{\infty} c_{k} \exp \left(i n \lambda_{k}\right), n \in \mathbb{Z}$, где коэффициенты $c_{k}, k \in \mathbb{N}$, определяются по формулам (32). По теореме $10 f(z)=\sum_{k=1}^{\infty} c_{k} \exp \left(i z \lambda_{k}\right), z \in \mathbb{C}$. Следовательно, имеет место (5). Теорема 1 доказана.

Приведем пример функции $\widehat{B}(\lambda)$, удовлетворяющей условиям теоремы 1 для случая $\rho^{*}=2, \sigma^{*}>1 / 2$. Положим $\widehat{B}(\lambda)=\sin (i \pi \lambda) / \lambda$. Тогда $\lambda_{k}^{\prime}=i k, \lambda_{k}^{\prime \prime}=-i k, k \in \mathbb{N}$; $\Omega_{1}=\Omega_{2}=\left\{e^{k}, k \in \mathbb{N}\right\}$. Нетрудно вычислить, что $\tau_{1}=\tau_{2}=1 / 2$. Следовательно, условия (3) и (4) принимают вид

$$
\lim _{k \rightarrow \infty} \frac{1}{k^{2}} \ln \left|\prod_{n \neq k}\left(1-e^{k-n}\right)\right|=\frac{1}{2}
$$

Последнее равенство выполняется. Действительно,

$$
\ln \left|\prod_{n \neq k}\left(1-e^{k-n}\right)\right|=\sum_{n=1}^{k-1} \ln \left(e^{k-n}-1\right)+\sum_{n=k+1}^{\infty} \ln \left(1-e^{k-n}\right) .
$$

Нетрудно показать, что

$$
\lim _{k \rightarrow \infty} \frac{1}{k^{2}} \sum_{n=1}^{k-1} \ln \left(e^{k-n}-1\right)=\frac{1}{2}, \quad \lim _{k \rightarrow \infty} \frac{1}{k^{2}} \sum_{n=k+1}^{\infty} \ln \left(1-e^{k-n}\right)=0 .
$$

\section{СПИСОК ЦИТИРОВАННОЙ ЛИТЕРАТУРЫ}

[1] А.Ф. Леонтьев, Обобщения рядов экспонент, Наука, М., 1981.

[2] Я.И. Хургин, В.П. Яковлев, Финитные функиии в физике и технике, Наука, М., 1971.

[3] С. В. Попенов, "О весовом пространстве функций, аналитических в неограниченной выпуклой области в $\mathbb{C}^{m}$ ", Матем. заметки, 40:3 (1986), 374-384.

[4] Б. Я. Левин, Распределение корней целых функиий, Гостехиздат, М., 1956. 
[5] И.Ф. Красичков-Терновский, "Однородное уравнение типа свертки на выпуклых областях", Докл. АН СССР, 197:1 (1971), 29-31.

[6] А. С. Кривошеев, В. В. Напалков, "Комплексный анализ и операторы свертки", УМН, 47:6 (1992), 3-58.

[7] R. Meise, K. Schwerdtfeger, B. A. Taylor, "On kernels of slowly decreasing convolution operators", Doğa Mat., 10:1 (1986), 176-197.

[8] В.В. Напалков, "О базисе в пространстве решений уравнений свертки", Матем. заметки, 43:1 (1988), 44-55.

[9] А.С. Кривошеев, "Базис Шаудера в пространстве решений однородного уравнения свертки", Матем. заметки, 57:1 (1995), 57-71.

[10] В. А. Ткаченко, "Спектральная теория в пространствах аналитических функционалов для операторов, порождаемых умножением на независимую переменную", Maтем. сб., 112:3 (1980), 421-466.

[11] М. М. Драгилев, В.П. Захарюта, Ю.Ф. Коробейник, “Двойственная связь между некоторыми вопросами теории базиса и интерполяции", Докл. АH CCCP, 215:3 (1974), 522-525.

[12] И.А. Шагапов, Инвариантные подпространства в пространствах числовых последовательностей, Дисс. ... к.ф.-м.н., ИМВЦ УНЦ РАН, Уфа, 1999.

[13] Себаштьян-и-Сильва, "О некоторых классах локально выпуклых пространств, важных в приложениях", Математика, 1:1 (1957), 60-77.

[14] А. И. Маркушевич, Теория аналитических функиий, 2, Наука, М., 1968.

[15] А. В. Братищев, Ю.Ф. Коробейник, "О некоторых характеристиках роста субгармонических функций", Матем. сб., 106:1 (1978), 44-65.

[16] Р. Эдвардс, Функционалъный анализ, Мир, М., 1969.

[17] А. В. Братищев, "Один тип оценок снизу целых функций конечного порядка и некоторые приложения", Изв. АН СССР. Сер. матем., 48:3 (1984), 451-475.

[18] А. П. Робертсон, В. Дж. Робертсон, Топологические векторные пространства, Мир, M., 1967.

\section{В. В. Напалков, В.Э. Ким}

Институт математики с вычислительным центром Уфимского научного центра Российской академии наук

E-mail: kim@imat.rb.ru
Поступило

28.05.2004

Исправленный вариант

02.12 .2005 